\title{
ANOMALY DETECTION PERFORMANCE COMPARISON ON ANOMALY- DETECTION BASED CHANGE DETECTION ON MARTIAN IMAGE PAIRS
}

\author{
Alfiah Rizky Diana Putri, Panagiotis Sidiropoulos, Jan-Peter Muller \\ Imaging Group, Mullard Space Science Laboratory, Dept. of Space and Climate Physics, \\ University College London, Holmbury St Mary, Surrey, RH5 6NT, UK \\ - (alfiah.putri.15, p.sidiropoulos, j.muller)@ucl.ac.
}

\section{Commission III, WG III/II}

KEY WORDS: change detection, Martian surface dynamics, mars, machine learning

\begin{abstract}
:
The surface of Mars has been imaged in visible wavelengths for more than 40 years since the first flyby image taken by Mariner 4 in 1964. With higher resolution from orbit from MOC-NA, HRSC, CTX, THEMIS, and HiRISE, changes can now be observed on high-resolution images from different instruments, including spiders (Piqueux et al., 2003) near the south pole and Recurring Slope Lineae (McEwen et al., 2011) observable in HiRISE resolution. With the huge amount of data and the small number of datasets available on Martian changes, semi-automatic or automatic methods are preferred to help narrow down surface change candidates over a large area.
\end{abstract}

To detect changes automatically in Martian images, we propose a method based on a denoising autoencoder to map the first Martian image to the second Martian image. Both images have been automatically coregistered and orthorectified using ACRO (Autocoregistration and Orthorectification) (Sidiropoulos and Muller, 2018) to the same base image, HRSC (High-Resolution Stereo Camera) (Neukum and Jaumann, 2004; Putri et al., 2018) and CTX (Context Camera) (Tao et al., 2018) orthorectified using their DTMs (Digital Terrain Models) to reduce the number of false positives caused by the difference in instruments and viewing conditions. Subtraction of the codes of the images are then inputted to an anomaly detector to look for change candidates. We compare different anomaly detection methods in our change detection pipeline: OneClassSVM, Isolation Forest, and, Gaussian Mixture Models in known areas of changes such as Nicholson Crater (dark slope streak), using image pairs from the same and different instruments.

\section{INTRODUCTION}

\subsection{Martian Surface Dynamics}

Over the last 40 years of Martian observations from orbit and from its surface, a large number of images has been obtained of the surface of Mars. The Martian surface has been previously thought to be static is very dynamic in certain regions, as there are more and more findings of Martian surface dynamics. These dynamic features vary from transient features such as new impact craters, features that are very small and can only be observed in $25 \mathrm{~cm} /$ pixel HiRISE images such as Recurring Slope Lineae (RSL) (McEwen, et al., 2011) that are suspected to be caused by water movement, features that are only found in the polar region such as Araneiform ("spiders") over the South Polar Residual Cap (SPRC), and a lot of others.

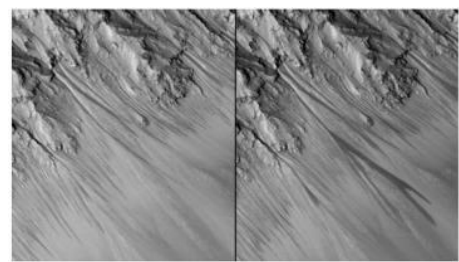

(a)

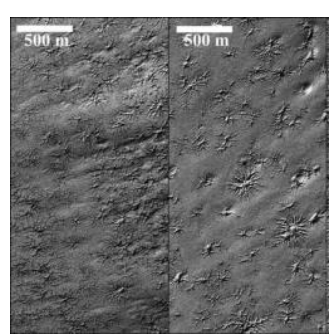

(b)

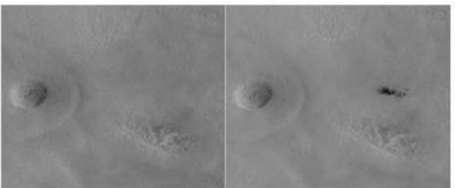

(c)

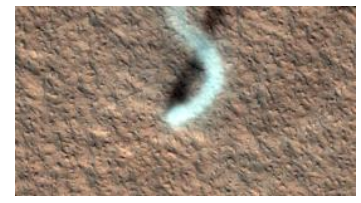

(d)
Figure 1. Examples of dynamic features on Martian surface: (a) Recurring Slope Lineae (RSL), (b) Araneiform ("spiders") (MOC) (Piqueux, 2003) (c) New impact craters (MOC) (d) Dust devil (HiRISE)

\subsection{Martian Change Detection Research}

Changes on Mars have been observed even before spacecraft were launched to Mars. Seasonal variations had been observed by Schiaparelli in the late 19th. With the aid of a telescope, regional-scale variations had been photographed. With 2 Martian Years (MY) of observations by Viking Orbiter, global changes were observed. With the higher resolution of Mars Orbiter Camera (MOC) Wide Angle (MOC-WA) and Narrow Angle (NA) camera, changes such as new impact craters and araneiform were observed. Improvement of change measurement could be done by the launch of higher-resolution cameras such as High-Resolution Stereo Camera (HRSC), Context Camera (CTX), and HIRISE, and topography of the area surrounding the features could be derived by the availability of Digital Terrain Model from Mars Orbital Laser Altimeter (MOLA), HRSC, CTX, and HiRISE. 
Automatic methods for Martian object detection have been more and more widely developed, such as for craters (Francis et al, 2019; Cohen et al., 2016, Bandeira, et al, 2007, amongst others) and for volcanic rootless cones (Palafox et al, 2017) and or features that are more dynamic in nature such as new impact craters (Xin et al., 2017) or dark slope streaks (Wang et al., 2017). Automatic change detection method by comparing temporal images however are not as well developed (Putri et al., 2018; Sidiropoulos and Muller, 2017; Di et al, 2014; Wagstaff et al, 2012)

With the huge amount of data and the small number of datasets available on Martian changes, semi-automatic or automatic methods are preferred to help narrow down change candidates over a large area.

\section{METHOD}

\subsection{Preprocessing}

Misregistration is an issue in change detection algorithms, especially in pixel-based methods. Previously Sidiropoulos and Muller (2018) developed ACRO based on SIFT (ScaleInvariant Feature Transform) and ring matching to automatically coregister and orthorectify high-resolution Martian images to the same base images from HRSC (Neukum and Jaumann, 2004) as HRSC has imaged $98 \%$ of Mars at 100 $\mathrm{m} /$ pixel or higher and always in stereo. Images are coregistered to individual HRSC images and when available to HRSC mosaics (Sidiropoulos and Muller, 2018; Putri et al., 2019). Where available, higher resolution images are coregistered to CTX images orthorectified to CTX DTMs (Tao et al., 2018).

\subsection{Feature Extraction using Denoising Autoencoder}

Another problem for change detection algorithms in planetary images using images obtained from different cameras are the different imaging conditions. With 4-layer denoising autoencoders with $345 \mathrm{~m} \times 345 \mathrm{~m}(64 \times 64)$ normalised CTX (single-band) inputs with a 32-pixel stride, the first image is mapped to the second image automatically as a way to encode the effect of the different viewing condition.

\subsection{Anomaly Detector}

As the second image has been mapped from the first image, the difference between the first image to the second image caused by the viewing conditions is usually known. Anomaly detection or commonly called outlier detection or novelty detection is used to obtain information about unpredicted change.

In this research we are comparing the performance of three different anomaly detection methods OneClassSVM, Isolation Forest, and Gaussian Mixture Models (GMM). OneClassSVM is an unsupervised SVM (support vector machine) trained to only one class of data, the "normal" data. After trained, the classifier could define input data not following the model as "anomalies". Isolation forest uses decision-tree and works by randomly selects a feature and a split value between the maximum and minimum values of the feature. "Normal" sample will need more conditions to be separated with other samples, different with an "anomaly", which is easily "isolated". GMM is using gaussian probabilistic model to divide data to subpopulations, of which small and unusual populations are "anomalies".
Figure 3 explains the steps from 2.1, 2.2, and 2.3 schematically.

\subsection{Change Dataset}

To test the method, Martian image pairs from different highresolution images are used to test the anomaly detection method. To test whether the method is able to perform well in unsupervised conditions, we annotated different image pairs from the same and different 5 test areas with different surface changes as there is currently only a few change detection datasets available on planetary surface.

Changes are annotated manually in QGIS as vectors to obtain georeferenced information of changes available to overlapping images at different resolutions. The change vector information is then rasterised to each image from the image pair resolution using ENVI Vector to ROI tool that works faster than using gdal_rasterize function.
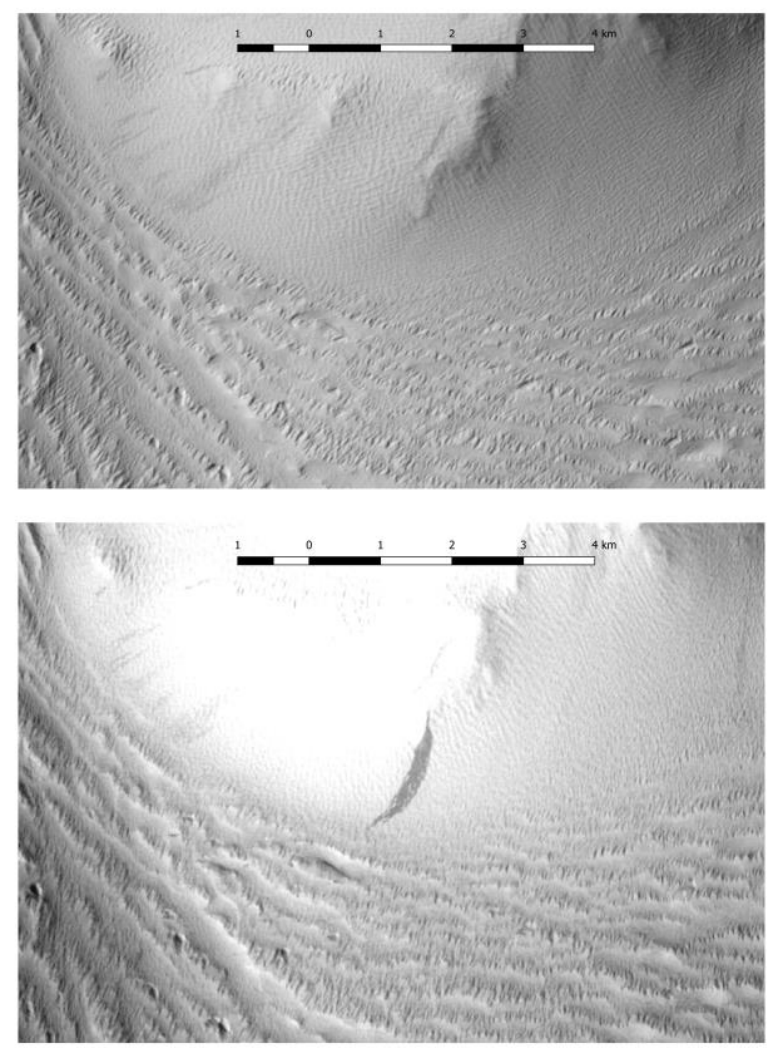

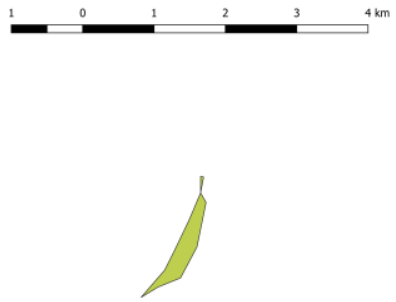

Figure 2. Example of a change and annotation done in this research centred in $194^{\circ} \mathrm{E}, 0^{\circ} \mathrm{N}$. Top image is

G17_024812_1801_XI_00N164W (11-11-2011), middle image is F09_039146_1801_XI_00N164W (02-12-2014), and the bottom image is annotation of the dark slope streak change

\subsection{Implementation}

Singular HRSC images are produced using NASA/DLRVICAR, while ACRO runs on MATLAB and C++ using ISIS3 for image ingestion. Autoencoder is implemented in Keras (Chollet, 2015) running on top of TensorFlow in Python and anomaly detector is based on Scikit.Learn . Algorithm is tested in $3.6 \mathrm{Ghz}$ i7-4790, 16GB RAM CPU, resulting in 700 s/epoch (Nicholson Crater) and 150s/ epoch (South Pole).

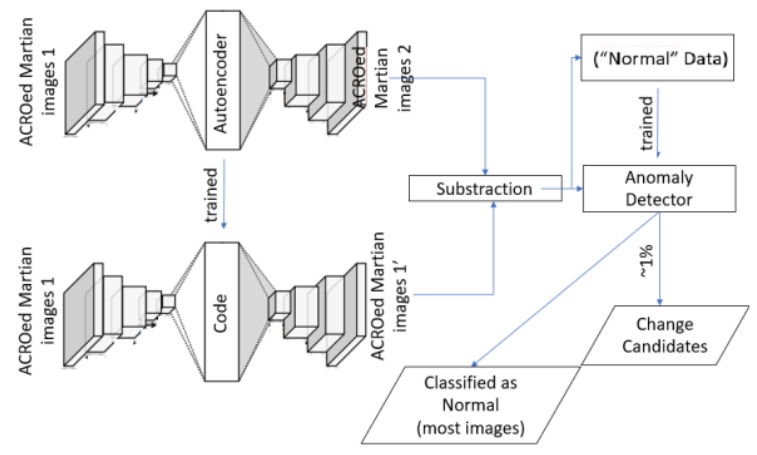

Figure 3. Schematic view of the overall change detection algorithm

\section{RESULTS}

In this research we compared the change detection result obtained by different anomaly detection method. Changes are detected when there are changed pixel in the image square. Table 1 shows the performance comparison of anomaly detection method in example case 1 dark slope streak in Nicholson Crater and Table 2 shows example. Table shows setting where high recall is desired.
Table 1 Performance of Anomaly Detection Method in Example Case 1 Dark Slope Streak in Nicholson Crater

\begin{tabular}{|c|c|c|c|c|}
\hline & OneClassSVM & $\begin{array}{c}\text { Isolation } \\
\text { Forest }\end{array}$ & $\begin{array}{c}\text { Gaussian } \\
\text { Mixture } \\
\text { Model } \\
\text { (no } \\
\text { known } \\
\text { change) }\end{array}$ & $\begin{array}{c}\text { Gaussian } \\
\text { Mixture } \\
\text { Model } \\
\text { (known } \\
\text { change) }\end{array}$ \\
\hline Accuracy & $99.55 \%$ & $96.6 \%$ & $52.89 \%$ & $94.08 \%$ \\
\hline Precision & $31.82 \%$ & $10.7 \%$ & $0.68 \%$ & $5.18 \%$ \\
\hline Recall & $100 \%$ & $87.93 \%$ & $100 \%$ & $100 \%$ \\
\hline F1 & $48.28 \%$ & $19.08 \%$ & $1.35 \%$ & $9.85 \%$ \\
\hline
\end{tabular}

Table 2 Performance of anomaly detection method in Example Case 2 Fans in South Polar Residual Cap

\begin{tabular}{|c|c|c|c|c|}
\hline & OneClassSVM & $\begin{array}{c}\text { Isolation } \\
\text { Forest }\end{array}$ & $\begin{array}{c}\text { Gaussian } \\
\text { Mixture } \\
\text { Model } \\
\text { (no } \\
\text { known } \\
\text { change) }\end{array}$ & $\begin{array}{c}\text { Gaussian } \\
\text { Mixture } \\
\text { Model } \\
\text { (known } \\
\text { change) }\end{array}$ \\
\hline Accuracy & $6.04 \%$ & $75.73 \%$ & $74.20 \%$ & $96.99 \%$ \\
\hline Precision & $14.79 \%$ & $16.94 \%$ & $27.94 \%$ & $76.86 \%$ \\
\hline Recall & $98.34 \%$ & $86.99 \%$ & $100 \%$ & $100 \%$ \\
\hline F1 & $25.71 \%$ & $28.36 \%$ & $43.68 \%$ & $86.92 \%$ \\
\hline
\end{tabular}

Based on the result in Table 1 and Table 2, we could see that OneClassSVM performs well in non-polar areas while obtained very low accuracy in the polar region because of the high number of changes found in the polar region resulting in higher anomaly threshold needed to obtain better recall. Isolation Forest works as well as OneClassSVM in non-polar areas, but performs better than OneClassSVM in polar area in terms of accuracy. Gaussian Mixture Model (GMM) uses probabilistic model to find subpopulation on data. Without knowing change subpopulations, GMM doesn't work as well in non-polar region, but works rather well in the polar region. By knowing one change example, subpopulation of change can be narrowed down, obtaining very good result in precision for our polar example.

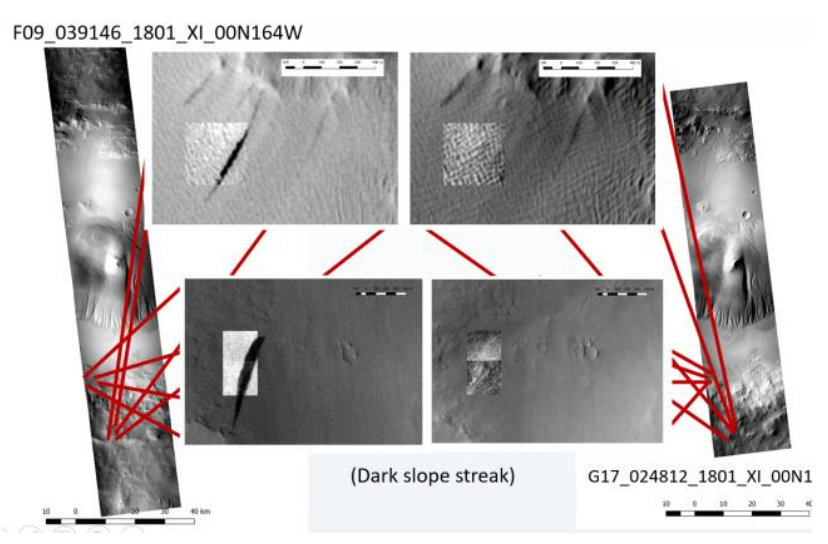

Figure 4. Example of the performance of the anomaly detection method (OneClassSVM) to detect dark slope streak changes between two CTX images 


\section{SUMMARY AND FUTURE WORK}

\subsection{Summary}

This paper has discussed a performance comparison of different anomaly detection methods in the anomaly detection-based change detection on Martian images. The pair of multiinstrument images first coregistered to the same base images, then converted to the resolution of the coarser image of the pair. The first image then mapped to the second image using a trained autoencoder. The difference between encoded second image and the second image then used as inputs for anomaly classifier

Based on their performance, Gaussian Mixture Models work the best in the polar region and obtain the highest recall value while OneClassSVM and Isolation Forest performs similarly well in non-polar region, while Isolation Forest performs better overall without change knowledge while GMM works the best if an example change is known.

\subsection{Future Work}

With the comparison of the performance of the anomaly detection methods in our change cases, we could generalise the characteristics of our method. Based on the result we obtained, we set our threshold to obtain a very high recall but quite low precision to obtain all changes and narrow down change candidates. Different classifier threshold can be fine-tuned to obtain higher precision or balancing between desired precision and recall. Based on the current result, precision can be increased as well.

Knowing the characteristics of our method, we could use our method in different areas to obtain candidates of change in larger region (in the polar region, especially the Martian south) and in a specific area encompassing the available (in Nicholson crater and Noctis Labyrinthus) to help change detection research on Mars.

\section{ACKNOWLEDGEMENTS}

Part of the research leading to these results has received partial funding from the European Union's Seventh Framework Programme (FP7/2007-2013) under iMars grant agreement $n^{\circ}$ 607379; MSSL STFC Consolidated grant no. ST/K000977/1 and the first author is supported by the Indonesian Endowment Fund for Education.

\section{REFERENCES}

Bandeira, L., Ding, W. and Stepinski, T.F., 2012. Detection Of Sub-Kilometer Craters in High Resolution Planetary Images Using Shape and Texture Features. Advances in Space Research, 49(1), pp.64-74.

Chollet, F., et al. 2015. Keras. Github: https://github.com/fchollet/keras.

Cohen, J.P., Lo, H.Z., Lu, T. and Ding, W., 2016. Crater Detection via Convolutional Neural Networks. arXiv preprint arXiv:1601.00978.
Di, K., Liu, Y., Hu, W., Yue, Z. and Liu, Z., 2014. Mars Surface Change Detection from Multi-Temporal Orbital Images. In IOP Conference Series: Earth and Environmental Science (Vol. 17, No. 1, p. 012015). IOP Publishing.

Francis, A., Muller, J.P., Sidiropoulos, P. and Persaud, D., 2018, September. Detection of Sub-Km Craters on Mars for Equilibrium Population Statistics. In European Planetary Science Congress (Vol. 12).

McEwen, A.S., Ojha, L., Dundas, C.M., Mattson, S.S., Byrne, S., Wray, J.J., Cull, S.C., Murchie, S.L., Thomas, N. and Gulick, V.C., 2011. Seasonal Flows on Warm Martian Slopes. Science, 333(6043), pp.740-743.

Neukum, G. and Jaumann, R., 2004, August. HRSC: The High Resolution Stereo Camera of Mars Express. In Mars Express: The Scientific Payload (Vol. 1240, pp. 17-35).

Palafox, L.F., Hamilton, C.W., Scheidt, S.P. and Alvarez, A.M., 2017. Automated Detection of Geological Landforms on Mars using Convolutional Neural Networks. Computers \& geosciences, 101, pp.48-56.

Pedregosa, F. and Varoquaux, G. and Gramfort, A. and Michel, V. and Thirion, B. and Grisel, O. and Blondel, M. and Prettenhofer, P. and Weiss, R. and Dubourg, V. and Vanderplas, J. and Passos, A. and Cournapeau, D. and Brucher, M. and Perrot, M. and Duchesnay, E.. 2011.Scikit-learn: Machine Learning in Python Journal of Machine Learning Research 12, pp. 2825-2830, 2011.

Piqueux, S., Byrne, S. and Richardson, M.I., 2003. Sublimation of Mars's Southern Seasonal $\mathrm{CO}_{2}$ Ice Cap and The Formation of spiders. Journal of Geophysical Research: Planets, 108(E8).

Putri, A.R.D., Sidiropoulos, P. and Muller, J.P., 2018, September. Deep Learning-Based Anomaly Detection to Find Changes over the Martian South Pole. In European Planetary Science Congress (Vol. 12).

Putri, A.R.D., Sidiropoulos, P., Muller, J.P., Walter, S.H. and Michael, G.G., 2019. A New South Polar Digital Terrain Model of Mars from the High-Resolution Stereo Camera (HRSC) onboard the ESA Mars Express. Planetary and Space Science. https://doi.org/10.1016/j.pss.2019.02.010

Sidiropoulos, P. and Muller, J.P., 2017, April. Automatic Detection of Changes on Mars Surface from High-Resolution Orbital Images. In EGU General Assembly Conference Abstracts (Vol. 19, p. 18300).

Sidiropoulos, P. and Muller, J.P., 2018. A Systematic Solution to Multi-Instrument Coregistration of High-Resolution Planetary Images to an Orthorectified Baseline. IEEE Transactions on Geoscience and Remote Sensing, 56(1), pp.7892.

Tao, Y., Muller, J.P., Sidiropoulos, P., Xiong, S.T., Putri, A.R.D., Walter, S.H.G., Veitch-Michaelis, J. and Yershov, V., 2018. Massive Stereo-Based DTM Production for Mars on Cloud Computers. Planetary and Space Science, 154, pp.30-58.

Wagstaff, K.L., Panetta, J., Ansar, A., Greeley, R., Pendleton Hoffer, M., Bunte, M. and Schörghofer, N., 2012. Dynamic Landmarking for Surface Feature Identification and Change 
Detection. ACM Transactions on Intelligent Systems and Technology (TIST), 3(3), p.49.

Wang, Y., Di, K., Xin, X. and Wan, W., 2017. Automatic Detection of Martian Dark Slope Streaks by Machine Learning using HiRISE Images. ISPRS Journal of Photogrammetry and Remote Sensing, 129, pp.12-20.

Xin, X., Di, K., Wang, Y., Wan, W. and Yue, Z., 2017. Automated Detection of New Impact Sites on Martian Surface from HiRISE Images. Advances in Space Research, 60(7), pp.1557-1569. 